\title{
Scalp Nerve Block Using Bupivacaine or Levobupivacaine Provides Effective and Safe Acute Pain Control to Pediatric Patients underwent Surgery for Moyamoya Disease
}

\author{
Cheng, Hsiao-Liang M.D. ( b87401032@ntu.edu.tw ) \\ 1. Department of Anesthesiology, National Taiwan University Hospital and National Taiwan University Medical College, Taipei, Taiwan \\ 2. Graduate Institute of Biomedical Electronics and Bioinformatics, College of Electrical Engineering and Computer Science, National \\ Taiwan University, Taipei, Taiwan
}

\section{Background:}

Regional scalp nerve block is an old but very useful technique in brain surgery. It includes the local anesthetics injection to supratrochlear and supraorbital nerves, zygomaticotemporal nerve, auriculotemporal nerve, and lesser occipital, greater occipital nerves. Combined with general anesthesia, it provides good blood pressure control, as well as satisfying pain management.

During many brain surgeries, the cranial fixation causes the most significant hemodynamic change, even more severe than incision to the scalp, thus intravenous analgesics or antihypertensive agents are required. If scalp nerve block is applied, the hemodynamics will be more stable and the pain control will be better during postop recovery.

Most of previous studies and previous work of our team included adults only. In this study, we suppose scalp nerve block provides similar benefit to pediatric patients as well.


\section{Materials and Methods:}

Our study includes all consecutive patients suffering from moyamoya disease underwent elective surgery during 2015/11 and 2016/10 and the exclusion criteria are emergent surgery, patients elder than 18 years old, or any contraindication of nerve block such as local infection or allergy. The anesthesia induction agents were fentanyl $(2-3 \mathrm{mcg} / \mathrm{Kg})$, propofol $(2-3 \mathrm{mg} / \mathrm{Kg})$ or thiamylal $(4-6 \mathrm{mg} / \mathrm{Kg})$ and cisatracurium $(0.15-0.2 \mathrm{mg} / \mathrm{Kg})$ or rocuronium $(0.8-1.0 \mathrm{mg} / \mathrm{Kg})$, followed by sevoflurane $(0.7-1.3$ $\mathrm{MAC}$ ) or desflurane (0.7-1.3 MAC) or propofol (TCI Ce 3.5-5.5 $\mathrm{mcg} / \mathrm{ml}$ ) for maintainance. If regional scalp nerve block was performed, the local anesthetics were $0.5 \%$ levobupivacaine or bupivacaine, and we injected each side for supratrochlear, supraorbital, zygomaticotemporal, greater and lesser occipital nerve after general anesthesia induction. We do not perform auriculotemporal nerve block to avoid possible damage to temporal artery which is critically important in moyamoya disease patients. Pain scores and analgesic medications during the first 24 hours after surgery were reviewed to assess the efficacy of nerve block.

\begin{tabular}{|l|l|l|l|l|l|l|l|l|}
\hline Age & & local analgesics & A & NP & I & K & NB & U \\
\hline $6 y 6 m$ & F & nil & 5 & 0 & 0 & 0 & 0 & 0 \\
\hline $3 y 8 m$ & F & nil & 4 & 0 & 0 & 0 & 0 & 0 \\
\hline $6 y 9 m$ & F & nil & 3 & 0 & 0 & 0 & 2 & 3 \\
\hline $9 y 8 m$ & M & nil & 3 & 0 & 0 & 0 & 0 & 0 \\
\hline $17 y 6 m$ & F & nil & 3 & 3 & 0 & 1 & 1 & 0 \\
\hline $10 y 5 m$ & M & nil & 4 & 0 & 0 & 0 & 0 & 0 \\
\hline & & & & & & & & \\
\hline $13 y 8 m$ & M & levobupivacaine & 0 & 4 & 0 & 0 & 0 & 0 \\
\hline $11 y 3 m$ & M & bupivacaine & 4 & 0 & 0 & 0 & 0 & 0 \\
\hline $8 y 6 m$ & M & bupivacaine & 4 & 0 & 0 & 0 & 0 & 0 \\
\hline $6 y 10 m$ & M & levobupivacaine & 4 & 0 & 0 & 0 & 0 & 0 \\
\hline $7 y 1 m$ & F & levobupivacaine & 3 & 0 & 4 & 0 & 0 & 0 \\
\hline
\end{tabular}

A: acetaminophen, NP: naproxen, I: ibuprofen, K: ketorolac, NB: nalbuphine, U: acetaminophen + tramadol

\section{Results:}

The total number of surgery for moyamoya disease during these twelve months were 16, and 11 of them were children under age 18. Surgeries included EDAS, EPS, or combination of them. Five patients received regional scalp nerve block and six didn't. All patients were extubated in OR and sent to ICU for intensive care at least one night. In the patients without scalp nerve block, the analgesics used were Acetaminophen 3.67, Naproxen 0.5, Ketorolac 0.167, Nalbuphine 0.5, Ultracet 0.5 (all showed as "average given times/ $24 \mathrm{hrs}$ "). In the patients with scalp nerve block, the analgesics used were Acetaminophen 3, Naproxen 0.8 , Ibuprofen 0.8 (all showed as "average given times/ $24 \mathrm{hrs"}$ ). There was no adverse event caused by regional scalp nerve block in our study.

\section{Conclusion:}

In pediatric patients with regional scalp nerve block to supratrochlear, supraorbital, zygomaticotemporal, greater and lesser occipital nerve, the pain control is better than patients without regional scalp nerve block. The analgesic medication in the first 24 hours after surgery were reduced. It is an effective and safe technique for moyamoya disease surgery.

References:

1. Regional scalp block for postcraniotomy analgesia: a systematic review and meta-analysis. Guilfoyle MR, Helmy A, Duane D, Hutchinson PJ. Anesth Analg. 2013 May;116(5):1093102.

2. 2. A review of scalp blockade for cranial surgery. Papangelou A, Radzik BR, Smith T, Gottschalk A. J Clin Anesth. 2013 Mar;25(2):150-9.

3. Effect of scalp blocks with levobupivacaine on recovery profiles after craniotomy for aneurysm clipping: a randomized, double-blind, and controlled study. Hwang JY, Bang JS, Oh CW, Joo JD, Park SJ, Do SH, Yoo YJ, Ryu JH. World Neurosurg. 2015 Jan;83(1):10813.

4. The effect of scalp block and local infiltration on the haemodynamic and stress response to skull-pin placement for craniotomy. Geze S, Yilmaz AA, Tuzuner F. Eur J Anaesthesiol. 2009 Apr;26(4):298-303

. Multiple combined indirect procedure for the surgical treatment of children with moyamoya disease. A comparison with single indirect anastomosis and direct anastomosis. Matsushima T, Inoue T, Ikezaki K, Matsukado K, Natori Y, Inamura T, Fukui M. Neurosurg Focus. 1998 Nov 15;5(5):e4. 